\title{
CR MANIFOLDS WITH NONCOMPACT CONNECTED AUTOMORPHISM GROUPS
}

\author{
JOHN M. LEE
}

\begin{abstract}
The main result of this paper is that the identity component of the automorphism group of a compact, connected, strictly pseudoconvex CR manifold is compact unless the manifold is $\mathrm{CR}$ equivalent to the standard sphere. In dimensions greater than 3, it has been pointed out by D. Burns that this result follows from known results on biholomorphism groups of complex manifolds with boundary and the fact that any such CR manifold $M$ can be realized as the boundary of an analytic variety. When $M$ is 3-dimensional, Burns's proof breaks down because abstract CR 3-manifolds are generically not realizable as boundaries. This paper provides an intrinsic proof of compactness that works in any dimension.
\end{abstract}

\section{INTRODUCTION}

The purpose of this paper is to prove the following theorem.

Theorem A. Let $M$ be a compact, connected, strictly pseudoconvex $C R$ manifold of dimension $2 n+1 \geq 3$. Then the identity component $\mathcal{A}(\mathcal{M})$ of the group $\mathcal{A}(\mathcal{M})$ of $C R$ automorphisms of $M$ is compact unless $M$ is globally $C R$ equivalent to the $(2 n+1)$-sphere with its standard $C R$ structure.

In dimensions greater than 3 , this result follows from known results on biholomorphism groups of complex manifolds with boundary. In fact, a stronger result is true in that case. The following is due to Dan Burns, although it has never been published:

Theorem 1.1. (D. Burns) Suppose $M$ is a compact, connected, strictly pseudoconvex $C R$ manifold of dimension $2 n+1 \geq 5$. The full $C R$ automorphism group $\mathcal{A}(\mathcal{M})$ is compact unless $M$ is globally $C R$ equivalent to $S^{2 n+1}$ with its standard $C R$ structure.

1991 Mathematics Subject Classification. Primary 32F40; Secondary 32C16, 32M99.

Research supported in part by National Science Foundation grant DMS 91-01832. 
The idea is that any such $M$ can be realized as the boundary of an analytic variety whose biholomorphism group is isomorphic to the group of CR automorphisms of $M$. It follows from results of B. Wong WO, J.-P. Rosay $\mathbb{R}$, and D. Burns and S. Shnider [BS (with a slight extra argument to deal with singular varieties) that any such variety with noncompact biholomorphism group is biholomorphic to the unit ball, so $M$ must be the sphere. Theorem 1.1 does not appear explicitly in the literature, so for completeness we give a sketch of Burns's proof in Section 2.

When $M$ is 3-dimensional, this proof breaks down because abstract CR 3-manifolds are generically not realizable as boundaries. The best previously known result is the following theorem of S. Webster. We say that a CR manifold is locally spherical if it is locally CR equivalent to the sphere with its standard CR structure.

Theorem 1.2. (S. Webster We2]) If $M$ is a compact, connected, strictly pseudoconvex $C R$ manifold, and $\mathcal{A}(\mathcal{M})$ is noncompact, then $M$ is locally spherical.

To put these results into perspective, is it useful to consider conformal Riemannian geometry as a guide, since there is a strong analogy between conformal and CR geometry. The analogous result for conformal manifolds is the following theorem. Recall that a Lie group $G$ acts properly on a space $X$ if the map $G \times X \rightarrow X \times X$ given by $(g, x) \mapsto(g \cdot x, x)$ is proper.

Theorem 1.3. Let $X$ denote a Riemannian manifold, $\mathrm{e}(X)$ its group of conformal diffeomorphisms, and $\mathrm{C}(X)$ the identity component of $\mathrm{e}(X)$

1. (M. Obata [0], U. Pinkall, and J. Lafontaine $\| \mathrm{La}$ ) If $X$ is compact, then $\mathrm{C}(X)$ is compact unless $X$ is conformally equivalent to the sphere with its standard metric.

2. (J. Ferrand [F1]) The same is true with $\mathrm{C}(\mathcal{X})$ replaced by $\mathrm{C}(\mathcal{X})$.

3. (J. Ferrand [F2]) If $X$ is non-compact, then $\mathrm{C}(X)$ acts properly unless $X$ is conformally equivalent to $\mathbf{R}^{\mathbf{n}}$ with the Euclidean metric.

These results have an interesting history. Theorem 1.3(1) was originally claimed by Obata in [O], and is commonly attributed to him. However, that paper contained a gap, which was repaired in [La], based on an argument due to Pinkall. Theorem 1.3(3) was claimed by V. Alekseevskii [A1, A2]. However, the proof in [A1] apparently contains a serious error (Theorem 4 is false), so the result was in question until 
the appearance of [F2]. The expository paper Gut by R. Gutschera gives an excellent survey of results in the conformal category.

By analogy with the conformal case, it is reasonable to make the following conjecture.

Conjecture 1.4. If $M$ is a connected, strictly pseudoconvex CR manifold, $\mathcal{A}(\mathcal{M})$ acts properly unless $M$ is $C R$ equivalent to the sphere or the Heisenberg group with its standard CR structure.

In case $M$ is compact, properness of the action implies compactness of $\mathcal{A}(\mathcal{M})$, so this conjecture includes as a special case the conjecture that $\mathcal{A}(\mathcal{M})$ is compact when $M$ is a compact strictly pseudoconvex 3 -manifold other than the sphere. When $M$ is noncompact, one can show as in Gut that $\mathcal{A}(\mathcal{M})$ acts properly if and only if it preserves a pseudohermitian structure (see $\$ 2$ for definitions).

P. Pansu has recently pointed out $[\mathrm{P}]$ that Ferrand's methods can be extended to the $\mathrm{CR}$ case to show that $\mathcal{A}(\mathcal{M})$ is precompact in the $C^{0}$ topology. But that approach is not yet strong enough to prove the full strength of Theorem A or Theorem 1.1, because it is not known in general whether a nonconstant uniform limit of CR diffeomorphisms is a diffeomorphism.

The proof of Theorem $\mathrm{A}$ is carried out along the lines of the proof of Theorem 1.3(1). In fact, as Webster already observed in We2, all but one step of that proof goes through in the CR case with little difficulty. Webster showed the following:

Theorem 1.5. (S. Webster We2 If $M$ is a compact, connected, strictly pseudoconvex, locally spherical CR manifold, and there exists a closed, noncompact one-parameter subgroup $G_{1} \subset \mathcal{A}(\mathcal{M})$ with a fixed point, then $M$ is globally $C R$ equivalent to $S^{2 n+1}$.

(The hypothesis that $G_{1}$ is closed was omitted from the statement of the theorem in We2, but it is clearly necessary.) Thus the only tricky part of Theorem $\mathrm{A}$ is proving that a closed, noncompact 1-parameter subgroup of $\mathcal{A}(\mathcal{M})$ has a fixed point. The bulk of this paper is devoted to proving this fixed-point result, which we state as a separate theorem.

Theorem B. Let $M$ be a compact, connected, strictly pseudoconvex $C R$ manifold of dimension $2 n+1 \geq 3$, and $G_{1} \subset \mathcal{A}(\mathcal{M})$ a closed, noncompact one-parameter subgroup. Then $G_{1}$ has a fixed point.

The corresponding step in the conformal case [O] was trivial: if the infinitesimal generator $X$ of $G_{1}$ never vanishes, then one can rescale the metric so that $X$ has norm 1 , and then $G_{1}$ preserves the rescaled metric, so compactness follows easily. In the $\mathrm{CR}$ case, the analogous 
argument only allows us to conclude that $X$ is tangent to the contact bundle $H$ somewhere. To draw the stronger conclusion that $X$ vanishes somewhere, we must carefully analyze the set $S$ where $X$ is tangent to $H$. This turns out to be a smoothly embedded compact hypersurface, which carries a Riemannian metric preserved by $G_{1}$. This implies that any sequence of elements of $G_{1}$ has a subsequence that converges along $S$. A further calculation shows that convergence along $S$ entails convergence of the 2-jets, from which global convergence follows.

Since there is no extra work involved, the proof is carried out in all dimensions, thereby providing an independent (and considerably more elementary) proof of Theorem 1.1 in the special case when the identity component $\mathcal{A}(\mathcal{M})$ is noncompact.

Here is the proof of Theorem A, given Theorem B. Assuming that $M$ is as in the statement of Theorem $\mathrm{A}$ and $\mathcal{A}(\mathcal{M})$ is noncompact, Webster's Theorem 1.2 implies that $M$ is locally spherical. It follows from the properties of the Cartan connection constructed by S. S. Chern and J. K. Moser [CM] and standard results on $G$-structures that $\mathcal{A}(\mathcal{M})$ has a unique smooth manifold structure making it into a Lie transformation group. (In fact, the topology on $\mathcal{A}(\mathcal{M})$ can be taken to be that of $C^{2}$ convergence on $M$.) If $\mathcal{A}(\mathcal{M})$ is not compact, then by an old theorem of D. Montgomery and L. Zippin [MZ] $\mathcal{A}(\mathcal{M})$ has a closed one-parameter subgroup $G_{1}$ which is isomorphic to $\mathbf{R}$. By Theorem $\mathbf{B}$, $G_{1}$ has a fixed point. But then Theorem 1.5 implies that $M$ is globally CR equivalent to $S^{2 n+1}$. Thus the theorem is proved.

An important application of this compactness result is to the construction of local slices for the action of the contact diffeomorphism group on the set of CR structures on a 3-manifold. This is carried out in a joint paper with J.-H. Cheng [CD].

In Section 2, we introduce our notation and review some facts from the theory of CR and pseudohermitian manifolds. At the end of the section, we sketch the proof of Theorem 1.1. In Section 3, we prove the fixed point theorem, Theorem B.

I would like to thank all the people with whom I have had useful discussions about this work, especially Jih-Hsin Cheng, Dan Burns, Robert Gutschera, Lee Stout, and Lutz Bungart.

\section{BACKGROUND}

Throughout this paper, we use the notation and terminology of [ $[\mathrm{Le}]$ unless otherwise specified; we refer the reader there for basic notions of CR geometry not explained here. Suppose $M$ is a hypersurface-type CR manifold of dimension $2 n+1$. This means $M$ is endowed with a smooth $n$-dimensional complex subbundle $\mathcal{H} \subset \mathcal{T} \mathcal{M} \otimes \mathbf{C}$ which satisfies 
CR MANIFOLDS WITH NONCOMPACT CONNECTED AUTOMORPHISM GROUPS

$\mathcal{H} \cap \mathcal{H}=\{\}$ and which is formally integrable: $[\Gamma(\mathcal{H}), \Gamma(\mathcal{H})] \subset \Gamma(\mathcal{H})$. The real bundle $H=\operatorname{Re}(\mathcal{H} \oplus \overline{\mathcal{H}}) \subset \mathcal{T} \mathcal{M}$ carries a complex structure map $J: H \rightarrow H$ satisfying $J^{2}=-1$, and $\mathcal{H}$ is just the $i$-eigenspace of the complexification of $J$.

An orientation of $T M$ together with the orientation of $H$ induced by $J$ automatically induces an orientation on the annihilator of $H$ in $T^{*} M$, so if $M$ is orientable there exists a global real 1-form $\theta$ whose kernel at each point is $H$. Once such a form $\theta$ is chosen, the Levi form determined by $\theta$ is the symmetric bilinear form on $H$ defined by

$$
\langle V, W\rangle_{\theta}=d \theta(V, J W) .
$$

The same formula, extended by complex bilinearity, gives a complexbilinear form on $H \otimes \mathbf{C}$ which is Hermitian on $\mathcal{H} \times \overline{\mathcal{H}}$. A standard computation shows that the Levi form changes conformally if $\theta$ is changed, so its signature is a CR invariant of $M$. If the Levi form is positive definite, the CR structure is said to be strictly pseudoconvex, and in that case $\theta$ is a contact form. A strictly pseudoconvex CR structure together with a given contact form is called a pseudohermitian structure.

On a pseudohermitian manifold, the Levi form yields a norm on all (real or complex) tensor bundles over $H$, denoted $|\cdot|_{\theta}$, and a characteristic vector field $T$, defined by

$$
T\lrcorner \theta=1, \quad T\lrcorner d \theta=0 .
$$

There is also a natural linear connection, the pseudohermitian connection We1, We3, T], and a natural Riemannian metric $g_{\theta}$ called the Webster metric We3], characterized as the unique inner product on $T M$ that restricts to the Levi form on $H$, and for which $T$ is a unit vector orthogonal to $H$. If we extend $J$ to an endomorphism $J_{\theta}$ of $T M$ by declaring $J_{\theta} T=0$, then $g_{\theta}$ can be written

$$
g_{\theta}(X, Y)=d \theta\left(X, J_{\theta} Y\right)+\theta(X) \theta(Y) .
$$

For local computations on a pseudohermitian manifold, it is useful to choose a complex local frame $\left(T, Z_{\alpha}, Z_{\bar{\alpha}}\right)$, where $\left\{Z_{\alpha}: \alpha=1, \ldots, n\right\}$ forms a basis for $\mathcal{H}$ at each point, $Z_{\bar{\alpha}}=\overline{Z_{\alpha}}$, and $T$ is the characteristic vector field of $\theta$. We let $\left\{\theta, \theta^{\alpha}, \theta^{\bar{\alpha}}\right\}$ denote the dual coframe. The components of a tensor with respect to this frame are denoted by subscript and/or superscript indices using the summation convention, with a zero index referring to the $T$ direction. For example, if $\eta$ is a 1-form, we can write locally

$$
\eta=\eta_{0} \theta+\eta_{\alpha} \theta^{\alpha}+\eta_{\bar{\alpha}} \theta^{\bar{\alpha}}
$$


The components of the Levi form in such a local frame are $h_{\alpha \bar{\beta}}$, where

$$
d \theta=i h_{\alpha \bar{\beta}} \theta^{\alpha} \wedge \theta^{\bar{\beta}} .
$$

We use the Hermitian matrix $h_{\alpha \bar{\beta}}$ and its inverse $h^{\alpha \bar{\beta}}$ to lower and raise indices in the usual way. The pseudohermitian connection is determined by a matrix of complex-valued 1-forms $\omega_{\beta}{ }^{\alpha}$ satisfying

$$
d \theta^{\alpha}=\theta^{\beta} \wedge \omega_{\beta}{ }^{\alpha}+A^{\alpha}{ }_{\bar{\beta}} \theta \wedge \theta^{\bar{\beta}},
$$

where $A^{\alpha}{ }_{\bar{\beta}}$ are the components of the Webster torsion tensor. The pseudohermitian curvature tensor is denoted by $R_{\alpha}{ }^{\rho} \beta \bar{\gamma}$, and the pseudohermitian Ricci tensor by $R_{\alpha \bar{\gamma}}=R_{\alpha}^{\beta} \beta \bar{\gamma}$.

Components of covariant derivatives are indicated with indices preceded by a semicolon. The most important commutation relations for covariant derivatives are the following (cf. [Le]): if $f$ is a smooth function on $M$, then

$$
\begin{gathered}
f_{; \alpha \bar{\beta}}-f_{; \bar{\beta} \alpha}=i h_{\alpha \bar{\beta}} f_{; 0} ; \quad f_{; 0 \alpha}-f_{; \alpha 0}=A_{\alpha \beta} f^{; \beta} ; \\
f_{; \alpha \beta \bar{\gamma}}-f_{; \alpha \bar{\gamma} \beta}=i h_{\beta \bar{\gamma}} f_{; \alpha 0}+R_{\alpha}{ }^{{ }{ }_{\beta \bar{\gamma}} f_{; \rho} .}
\end{gathered}
$$

For a smooth function $f, \bar{\partial}_{b} f$ is the restriction to $\overline{\mathcal{H}}$ of $d f$. On a pseudohermitian manifold, we can identify $\bar{\partial}_{b} f$ as an honest differential form by stipulating that $T\lrcorner \bar{\partial}_{b} f=0$, so in terms of a local frame we have

$$
\bar{\partial}_{b} f=f_{; \bar{\alpha}} \theta^{\bar{\alpha}} .
$$

The norm of $\bar{\partial}_{b} f$ is $\left|\bar{\partial}_{b} f\right|_{\theta}^{2}=f_{; \bar{\alpha}} f ; \bar{\alpha}$.

A real vector field $X$ on $M$ whose flow acts by CR automorphisms is called a $C R$ vector field. In particular, since the flow preserves the contact bundle $H$, any such vector field is an infinitesimal contact automorphism. It is well known (cf. Gr, CD]) that any such vector field is of the form

$$
X=H_{f}^{\theta}-f T
$$

for some real-valued smooth function $f$, where $H_{f}^{\theta}$ is the contact Hamiltonian field of $f$, defined by $\left.\left.H_{f}^{\theta}\right\lrcorner \theta=0, H_{f}^{\theta}\right\lrcorner d \theta=d f-(T f) \theta$. In terms of a local frame,

$$
H_{f}^{\theta}=i f^{; \bar{\alpha}} Z_{\bar{\alpha}}-i f^{; \alpha} Z_{\alpha} .
$$

If $X$ is a CR vector field, then in addition $X$ satisfies

$$
L_{X} \theta^{\bar{\beta}} \equiv 0 \quad\left(\bmod \theta, \theta^{\bar{\alpha}}\right)
$$

which implies (using 2.2)

$$
0=\left(L_{X} \theta^{\bar{\beta}}\right)\left(Z_{\alpha}\right)=i f^{; \bar{\beta}_{\alpha}}-A^{\bar{\beta}}{ }_{\alpha} f,
$$


CR MANIFOLDS WITH NONCOMPACT CONNECTED AUTOMORPHISM GROUP\$

or, lowering indices,

$$
f_{; \beta \alpha}=-i A_{\beta \alpha} f \text {. }
$$

We conclude this section by sketching a proof of Theorem 1.1. Let $M$ be as in the statement of the theorem. By Boutet de Monvel's embedding theorem [BdM], there exist smooth global CR-holomorphic functions $\left(z^{1}, \ldots, z^{m}\right)$ on $M$ that define a $\mathrm{CR}$ embedding of $M$ into $\mathbf{C}^{\mathbf{m}}$ for some $m$. Then by the "filling-in" theorem of R. Harvey and B. Lawson [H], $M$ bounds a unique compact complex analytic variety $V \subset \mathbf{C}^{\mathbf{m}}$, which is smooth except possibly at a finite set $\mathcal{S}$ of isolated singular points in its interior.

Let $\pi: \widehat{V} \rightarrow V$ denote the normalization of $V$ [G2]; thus $\widehat{V}$ is an abstract normal Stein analytic space with a finite singular set $\widehat{\mathcal{S}} \subset$ $\pi^{-1}(\mathcal{S})$, and $\pi$ is a biholomorphism on the complement of the finite set $\pi^{-1}(\mathcal{S})$. It follows that $\widehat{V}$ also has $M$ as smooth boundary.

Let $\mathcal{B}(\widehat{V})$ denote the group of biholomorphisms of $\widehat{V}$. Equipped with the compact-open topology, $\mathcal{B}(\widehat{\mathcal{V}})$ is a Lie group [Fu. I claim that $\mathcal{A}(\mathcal{M})$ and $\mathcal{B}(\widehat{\mathcal{V}})$ are topologically isomorphic. Let $\varphi \in \mathcal{A}(\mathcal{M})$ be any $\mathrm{CR}$ automorphism of $M$. Since $M=\partial V$ is strictly pseudoconvex, the coordinate functions of $\varphi: M \rightarrow M \subset \mathbf{C}^{\mathbf{m}}$ extend uniquely to a neighborhood of $M$ in $V$; then by Hartogs's theorem for Stein varieties GR, p. 228], $\varphi$ extends to a weakly holomorphic map $\Phi: V \rightarrow V$. By normality, the weakly holomorphic map $\Phi \circ \pi: \widehat{V} \rightarrow V$ extends holomorphically to all of $\widehat{V}$. Moreover, away from the proper subvariety $(\Phi \circ \pi)^{-1}(\mathcal{S})$, this map lifts to a map $\widehat{\Phi}=\pi^{-1} \circ \Phi \circ \pi: \widehat{V} \rightarrow \widehat{V}$, and by normality again, the lifted map $\widehat{\Phi}$ extends to all of $\widehat{V}$. Arguing similarly for $\varphi^{-1}$, we obtain a map $\widehat{\Psi}: \widehat{V} \rightarrow \widehat{V}$ such that $\widehat{\Psi} \circ \widehat{\Phi}$ and $\widehat{\Phi} \circ \widehat{\Psi}$ are the identity near the boundary of $\widehat{V}$, and by analytic continuation globally; thus $\widehat{\Phi}$ is a biholomorphism.

This construction yields a group homomorphism $E: \mathcal{A}(\mathcal{M}) \rightarrow \mathcal{B}(\widehat{\mathcal{V}})$. Fefferman's extension theorem [FE] (suitably localized near the boundary as in Fo ) shows that any biholomorphism $\widehat{\Phi}$ of $\widehat{V}$ extends smoothly to $M$, so $E$ is bijective. Since a Stein variety is holomorphically convex, convergence of $\left\{\varphi_{j}\right\} \subset \mathcal{A}(\mathcal{M})$ implies uniform convergence of $\left\{\widehat{\Phi}_{j}\right\}$, so $E$ is continuous. Since any continuous bijection between Lie groups is a diffeomorphism, it follows that $\mathcal{A}(\mathcal{M})$ and $\mathcal{B}(\widehat{\mathcal{V}})$ are smoothly isomorphic.

Suppose $\mathcal{A}(\mathcal{M})$ is noncompact. By the discussion above, $\mathcal{B}(\widehat{\mathcal{V}})$ is also noncompact. If we knew $\widehat{V}$ were smooth, noncompactness of $\mathcal{B}(\widehat{\mathcal{V}})$ would imply by [BS, Thm. II] (cf. also $[\mathrm{WO}, \mathbb{R}]$ ) that $\widehat{V}$ is biholomorphically equivalent to the $(n+1)$-ball. By Fefferman's theorem once 
again, the biholomorphism extends to a CR equivalence between $M$ and the sphere, thus proving the theorem. Thus to complete the proof it suffices to show that $\widehat{\mathcal{S}}$ is empty when $\mathcal{B}(\widehat{\mathcal{V}})$ is noncompact.

Suppose on the contrary that $\widehat{\mathcal{S}}$ is not empty and $\mathcal{B}(\widehat{\mathcal{V}})$ is noncompact. Then so is the subgroup $\mathcal{B}(\widehat{\mathcal{V}})$ consisting of biholomorphisms that fix $\widehat{\mathcal{S}}$ pointwise, since $\mathcal{B}(\widehat{\mathcal{V}})$ has finite index in $\mathcal{B}(\widehat{\mathcal{V}})$ due to the finiteness of $\widehat{\mathcal{S}}$. Let $\left\{\widehat{\Phi}_{j}\right\} \subset \mathcal{B}(\widehat{\mathcal{V}})$ be a sequence with no subsequence that converges in the compact-open topology to a biholomorphism. By applying Montel's theorem for varieties [G1, B] to the coordinate functions of $\pi \circ \widehat{\Phi}_{j}$, one can show that a subsequence converges uniformly on compact subsets to a holomorphic map $\widehat{\Phi}: \widehat{V} \rightarrow \widehat{V} \cup M$. Passing to a smaller subsequence and arguing similarly for $\widehat{\Phi}_{j}^{-1}$, we may assume also that $\widehat{\Phi}_{j}^{-1}$ converges to a holomorphic map $\widehat{\Psi}: \widehat{V} \rightarrow \widehat{V} \cup M$.

If $\widehat{\Phi}(p) \in M$ for some $p \in \widehat{V}$, then letting $f$ be a holomorphic peak function on $\widehat{V} \cup M$ taking its maximum modulus at $\Phi(p), f \circ \Phi$ would take an interior maximum at $p$, so would be constant by the maximum principle; but this contradicts the fact that each $\widehat{\Phi}_{j}$ fixes $\widehat{\mathcal{S}}$. Thus $\widehat{\Phi}$ maps $\widehat{V}$ to $\widehat{V}$; a similar comment applies to $\widehat{\Psi}$. Then uniform convergence of $\widehat{\Phi}_{j}$ and $\widehat{\Phi}_{j}^{-1}$ on a compact neighborhood of $\widehat{\mathcal{S}}$ implies $\widehat{\Psi} \circ \widehat{\Phi}$ and $\widehat{\Phi} \circ \widehat{\Psi}$ are the identity on an open set, and by analytic continuation globally, so $\widehat{\Phi}_{j}$ converges to a biholomorphism, which is a contradiction. This completes the proof.

\section{A FIXED-POINT THEOREM}

In this section we prove Theorem B, from which Theorem A follows as described in the Introduction.

We need to show that the infinitesimal generator $X$ of $G_{1}$ has a zero. The proof will be by contradiction. We begin by noting that we can assume without loss of generality that $M$ is orientable: if not, just lift the CR structure and $X$ to the orientable double cover, and the lifted vector field will have zeros if and only if $X$ does. When $M$ is orientable, it is possible to choose a global contact form $\theta$.

Before proving Theorem B, we establish some preliminary lemmas. Throughout this section, $M$ will be as in the statement of the theorem and $X$ will be the infinitesimal generator of $G_{1}$, with the additional assumptions that $M$ is orientable and $X$ has no zeros on $M$.

Lemma 3.1. There is a nonempty, compact, embedded hypersurface $S \subset M$ along which $X$ is tangent to both $H$ and $S$. 
CR MANIFOLDS WITH NONCOMPACT CONNECTED AUTOMORPHISM GROUPG

Proof. Choose a global contact form $\theta$ on $M$. Since $X$ is an infinitesimal contact diffeomorphism, we can write $X$ in the form (2.5), where $f=$ $-\theta(X)$ satisfies (2.7). If $f$ vanishes nowhere, then replacing $\theta$ by $\widetilde{\theta}=$ $(1 / f) \theta$, we see that $\widetilde{f}=-\widetilde{\theta}(X)=1$. This implies $H_{\tilde{f}}^{\tilde{\theta}}=0, X=-\widetilde{T}$, and therefore

$$
\left.\left.L_{X} \tilde{\theta}=-d(\widetilde{T}\lrcorner \widetilde{\theta}\right)-\widetilde{T}\right\lrcorner d \widetilde{\theta}=0
$$

which means that $G_{1}$ preserves the contact form $\tilde{\theta}$ and therefore the Riemannian metric $g_{\tilde{\theta}}$. Thus $G_{1}$ is contained in the isometry group $\left.\mathcal{J}(\}_{\tilde{\theta}}\right)$ of $g_{\tilde{\theta}}$, which is compact. Any sequence in $G_{1}$ therefore has a subsequence that converges in the topology of $\left.\mathcal{J}(\}_{\tilde{\theta}}\right)$, which implies uniform convergence with all derivatives, and hence also in the topology of $\mathcal{A}(\mathcal{M})$. Since $G_{1}$ is closed in $\mathcal{A}(\mathcal{M})$, the limit is again in $G_{1}$, so $G_{1}$ is compact, which is a contradiction. Thus $f$ must vanish somewhere on $M$.

Now let $S$ denote the zero set of $f$. In other words, $S$ is the set of points of $M$ where $X$ is tangent to $H$. At points of $S$, we have $X=H_{f}^{\theta}$, so the assumption that $X$ does not vanish means that $H_{f}^{\theta} \neq 0$ and therefore $d f \neq 0$ along $S$. Thus $S$ is a compact, embedded hypersurface in $M$. Moreover, since $H_{f}^{\theta}(f)=d f\left(H_{f}^{\theta}\right)=d \theta\left(H_{f}^{\theta}, H_{f}^{\theta}\right)=0, X$ is tangent to $S$.

Lemma 3.2. The contact form $\theta$ can be chosen so that $\left|\bar{\partial}_{b} f\right|_{\theta}=1$ along $S$. For any such $\theta$, the following relations hold along $S$.

$$
\begin{gathered}
f_{; \alpha \beta}=0 ; \\
f_{; 0}=0 ; \\
f^{; \beta} f_{; 0 \beta} \text { is real } ; \\
f_{; 0 \alpha}=f^{\beta} f_{; 0 \beta} f_{\alpha} .
\end{gathered}
$$

Proof. Since $H_{f}^{\theta} \neq 0$ at points of $S$, by rescaling $\theta$ we can guarantee that the Levi norm $\left|\bar{\partial}_{b} f\right|_{\theta}$ is equal to 1 along $S$, as follows. If $\tilde{\theta}=u \theta$ for some positive function $u \in C^{\infty}(M)$, then $\tilde{f}=-\widetilde{\theta}(X)=u f$, and so $\bar{\partial}_{b} \tilde{f}=u \bar{\partial}_{b} f$ along $S$. Therefore $\left|\bar{\partial}_{b} \widetilde{f}\right|_{\tilde{\theta}}^{2}=u^{-1}\left|u \bar{\partial}_{b} f\right|_{\theta}^{2}=u\left|\bar{\partial}_{b} f\right|_{\theta}^{2}$ along $S$, so replacing $\theta$ by $u \theta$, where $u$ is any positive function that equals $\left|\bar{\partial}_{b} f\right|_{\theta}^{-2}$ along $S$, gives a contact form satisfying the first statement of the lemma.

Let $\theta$ be any such contact form, and $f=-\theta(X)$. Since $X$ is a CR vector field, (2.7) evaluated along $S$ gives (3.8). 
Next we prove (3.9). Since $\left|\bar{\partial}_{b} f\right|_{\theta}^{2}=1$ on $S$ and $X$ is tangent to $S$, at points of $S$ we have

$$
\begin{aligned}
0 & =X\left|\bar{\partial}_{b} f\right|_{\theta}^{2}=i f^{; \bar{\alpha}}\left(f_{; \bar{\beta}} f^{; \bar{\beta}}\right)_{; \bar{\alpha}}-i f^{; \alpha}\left(f_{; \bar{\beta}} f^{; \bar{\beta}}\right)_{; \alpha} \\
& =i f^{; \bar{\alpha}} f_{; \bar{\beta}} f^{; \bar{\beta}}-i f^{; \alpha} f^{; \bar{\beta}} f_{; \bar{\beta} \alpha} \\
& =i f^{; \bar{\alpha}} f^{; \beta}\left(f_{; \beta \bar{\alpha}}-f_{; \bar{\alpha} \beta}\right) \\
& =i f^{; \bar{\alpha}} f^{; \beta}\left(i h_{\beta \bar{\alpha}} f_{; 0}\right)=-f_{; 0} .
\end{aligned}
$$

This implies that $T$ is also tangent to $S$. Therefore, on $S$ we have

$$
0=X T f=i f^{; \bar{\alpha}} f_{; 0 \bar{\alpha}}-i f^{; \alpha} f_{; 0 \alpha},
$$

which is (3.10).

Finally, since $f_{; 0}$ vanishes along $S$, we can write $f_{; 0}=v f$ for some smooth function $v$. Differentiating, we find that $f_{; 0 \alpha}=v f_{; \alpha}$ along $S$. Then contracting with $f^{; \alpha}$ yields $f_{; 0 \alpha} f^{; \alpha}=v f_{; \alpha} f^{; \alpha}=v$, which proves (3.11).

Lemma 3.3. The contact form $\theta$ can be chosen so that $L_{X} \theta=L_{X} d \theta=$ 0 at points of $S$.

Proof. Since $X$ is a contact vector field, $L_{X} \theta=-T f \theta$ on all of $M$. Therefore, by (3.9), $L_{X} \theta=0$ along $S$ provided $\theta$ is chosen as in Lemma 3.2. If $\widetilde{\theta}=v \theta$ is another contact form, then

$$
\begin{aligned}
L_{X} d \widetilde{\theta} & =d L_{X} \tilde{\theta} \\
& =d(X v \theta-v T f \theta) \\
& =d(X v) \wedge \theta+X v d \theta-v d(T f) \wedge \theta-T f d v \wedge \theta-v T f d \theta
\end{aligned}
$$

In order to preserve the property $\left|\bar{\partial}_{b} f\right|_{\theta}=1$ along $S$, we put $v=1+u f$ for some smooth function $u$ yet to be determined. Then we have the following relations at points of $S$ :

$$
\begin{gathered}
v=1 ; \\
X v=0 ; \\
d(X v)=u d(X f)+X u d f=u d(-f T f)+X u d f=X u d f .
\end{gathered}
$$

(In the last line we have used the fact that $H_{f}^{\theta} f=0$.) Therefore, along $S$ we have

$$
L_{X} d \widetilde{\theta}=X u d f \wedge \theta-d(T f) \wedge \theta .
$$

To prove the lemma, it suffices to produce a real-valued function $u$ such that $X u d f \equiv d(T f)(\bmod \theta)$ along $S$. In components this means, using (3.11),

$$
(X u) f_{; \alpha}=f_{; 0 \alpha}=f^{; \beta} f_{; 0 \beta} f_{; \alpha},
$$


CR MANIFOLDS WITH NONCOMPACT CONNECTED AUTOMORPHISM GROUP\$

which is equivalent to

$$
X u=f^{; \beta} f_{; 0 \beta} .
$$

The hypersurface $S$ is characteristic for this equation, so one might expect that existence of a global solution would depend on the global behavior of $f^{; \beta} f_{; 0 \beta}$ and the integral curves of $X$. Surprisingly, however, it turns out that we can write down an explicit solution.

To this end, consider the function $\Delta_{b} f=-\left(f_{; \beta} \beta+f_{; \bar{\beta}} \bar{\beta}\right)$. Since $f_{; \beta}{ }^{\beta}-f_{; \bar{\beta}} \bar{\beta}=n i f_{; 0}=0$ along $S$, we have the following at points of $S$ :

$$
\begin{aligned}
X\left(\Delta_{b} f\right) & =-2 X\left(f_{; \beta}{ }^{\beta}\right) \\
& =-2 i f^{; \bar{\alpha}} f_{; \beta}{ }^{\beta} \bar{\alpha}+2 i f^{; \alpha} f_{; \beta}{ }^{\beta}{ }_{\alpha} .
\end{aligned}
$$

Using the commutation relations (2.3) and (2.4), we can simplify the third derivatives that appear above as follows:

$$
\begin{aligned}
f_{; \beta}{ }^{\beta} \bar{\alpha} & =\left(f_{; \bar{\beta}} \bar{\beta}+n i f_{; 0}\right)_{; \bar{\alpha}} \\
& =f_{; \bar{\beta} \bar{\alpha}}{ }^{\bar{\beta}}+i \delta_{\bar{\alpha}}{ }^{\bar{\beta}} f_{; \bar{\beta} 0}-R_{\bar{\beta}} \overline{\bar{\rho}}{ }_{\bar{\alpha}}^{\bar{\beta}} f_{; \bar{\rho}}+n i f_{; 0 \bar{\alpha}} \\
& =\left(i A_{\bar{\beta} \bar{\alpha}} f\right)^{; \bar{\beta}}+i f_{; \bar{\alpha} 0}-R_{\rho \bar{\alpha}} f^{; \rho}+n i f_{; 0 \bar{\alpha}} \\
& =i A_{\bar{\beta} \bar{\alpha}} f^{; \bar{\beta}}+i f_{; \bar{\alpha} 0}-R_{\rho \bar{\alpha}} f^{; \rho}+n i f_{; 0 \bar{\alpha}} \\
& =(n+1) i f_{; 0 \bar{\alpha}}-R_{\rho \bar{\alpha}} f^{; \rho} . \\
f_{; \beta}{ }^{\beta} \alpha & =f_{; \beta \alpha}{ }^{\beta}-i \delta_{\alpha}{ }^{\beta} f_{; \beta 0}-R_{\beta}{ }^{\rho}{ }^{\beta} f_{; \rho} \\
& =\left(-i A_{\beta \alpha} f\right)^{; \beta}-i f_{; \alpha 0}-R_{\alpha \bar{\rho}} f^{; \bar{\rho}} \\
& =-i A_{\beta \alpha} f^{; \beta}-i f_{; \alpha 0}-R_{\alpha \bar{\rho}} f^{; \bar{\rho}} \\
& =-i f_{; 0 \alpha}-R_{\alpha \bar{\rho}} f^{; \bar{\rho}} .
\end{aligned}
$$

Inserting these relations into (3.12) and using (3.10), we obtain

$$
X\left(\Delta_{b} f\right)=2(n+1) f^{; \bar{\alpha}} f_{; 0 \bar{\alpha}}+2 f^{; \alpha} f_{; 0 \alpha}=2(n+2) f^{; \alpha} f_{; 0 \alpha} .
$$

Thus the conclusion of the lemma holds if we replace $\theta$ by $\widetilde{\theta}=(1+u f) \theta$, where $u$ is any function that is equal to $\Delta_{b} f /(2(n+2))$ along $S$.

Proof of Theorem B. Suppose $G_{1}$ has no fixed points, and let $\left\{\varphi_{j}\right\} \subset$ $G_{1}$ be any sequence; we will show it has a convergent subsequence, which is a contradiction.

If $\theta$ is chosen as in Lemma 3.3, the Webster metric $g_{\theta}=d \theta\left(\cdot, J_{\theta} \cdot\right)+\theta^{2}$ is preserved along $S$ by $G_{1}$, since $L_{X} \theta=L_{X} d \theta=L_{X} T=L_{X} J_{\theta}=0$ along $S$. Since the group of isometries of $\left.g_{\theta}\right|_{S}$ is compact, there is a subsequence, still denoted $\left\{\varphi_{j}\right\}$, whose restrictions to $S$ converge uniformly with all derivatives. 
I claim that the two-jets of the sequence $\left\{\varphi_{j}\right\}$ converge at all points of $S$. This implies that $\left\{\varphi_{j}\right\}$ converges in the topology of $\mathcal{A}(\mathcal{M})$ by the following standard argument. Let $Y \rightarrow M$ be the Chern CR structure bundle of $M$ [CM]; then every CR automorphism $\varphi$ of $M$ lifts naturally to an automorphism $\tilde{\varphi}$ of $Y$ preserving the Chern connection. Moreover, choosing any point $\xi \in Y$ over $S$, the orbit map $\varphi \mapsto \widetilde{\varphi}(\xi)$ gives a closed embedding of $G_{1}$ into $Y \llbracket \mathbb{K o}$, Thm. I.3.2]. Convergence of the two-jets of $\left\{\varphi_{j}\right\}$ along $S$ implies that $\left\{\widetilde{\varphi}_{j}(\xi)\right\}$ converges in $Y$, which therefore implies that $\left\{\varphi_{j}\right\}$ converges to some element $\varphi \in G_{1}$.

To see that convergence of $\varphi_{j}$ on $S$ implies convergence on the twojet level, let $Y$ denote the vector field $J_{\theta} X=J H_{f}^{\theta}$. Since $Y=f^{; \bar{\alpha}} Z_{\bar{\alpha}}+$ $f^{;}{ }^{; \alpha} Z_{\alpha}$ by (2.6), it follows that $Y f=2 f^{;} ; \bar{\alpha} f_{; \bar{\alpha}}=2$ along $S$. In particular, $Y$ is always transverse to $S$. For any element $\varphi \in G_{1}$, using the facts that $\varphi$ is a CR automorphism and $\varphi_{*} X=X$, we compute

$$
\begin{aligned}
\varphi_{*} Y & =\varphi_{*}\left(J H_{f}^{\theta}\right)=J\left(\varphi_{*} H_{f}^{\theta}\right) \\
& =J\left(\varphi_{*}(X+f T)\right)=J_{\theta} X+\left(f \circ \varphi^{-1}\right) J_{\theta}\left(\varphi_{*} T\right) \\
& =Y+\left(f \circ \varphi^{-1}\right) J_{\theta}\left(\varphi_{*} T\right) .
\end{aligned}
$$

Given a point $p \in S$, we can choose coordinates $\left(x_{1}, \ldots, x_{2 n}, y\right)$ on some neighborhood $U$ of $p$ such that $y=0$ on $S$ and $Y \equiv \partial / \partial y$. In these coordinates, $f(x, y)=2 y$, since both sides satisfy the ordinary differential equation $Y f=2$ with initial condition $f=0$ on $S$. Choosing analogous coordinates $(\widetilde{x}, \widetilde{y})$ near $\lim _{j \rightarrow \infty} \varphi_{j}(p) \in S$ and taking $j$ sufficiently large and $U$ sufficiently small, we may assume that $\varphi_{j}(x, 0)$ lies in a single coordinate chart for all $j$ and all $(x, 0) \in U \cap S$. In these coordinates, we can consider $\varphi_{j}$ as a vector-valued function of $(x, y)$. We already know that all the $x$-derivatives of $\varphi_{j}$ converge on $S$, so we need only consider the $y$-derivatives.

In coordinates, (3.13) becomes

$$
\frac{\partial \varphi_{j}(x, y)}{\partial y}=(0,1)+2 y V_{j}(x, y)
$$

where $V_{j}(x, y)$ is the coordinate representation of $J_{\theta}\left(\left(\varphi_{j}\right)_{*} T_{(x, y)}\right)$. In particular, along $S$ we get $\partial \varphi_{j} / \partial y \equiv(0,1)$, which certainly converges together with all its $x$-derivatives. Moreover, the values of $\partial^{2} \varphi_{j} / \partial y^{2}=$ $2 V_{j}=J_{\theta}\left(\left(\varphi_{j}\right)_{*} T\right)$ along $S$ are determined by the 1-jets of $\varphi_{j}$ along $S$, and we have already shown these converge on $S$. Therefore all second derivatives of $\varphi_{j}$ converge on $S$. This completes the proof of Theorem B. 
CR MANIFOLDS WITH NONCOMPACT CONNECTED AUTOMORPHISM GROURS

\section{REFERENCES}

[A1] D. V. Alexseevskii, Groups of conformal transformations of Riemannian spaces, Mat. Sbornik 89 (1972), in Russian; Math. USSR Sbornik 18 (1972) 285-301, in English.

[A2] $\quad S^{n}$ and $\mathbf{R}^{\mathbf{n}}$ are the only Riemannian manifolds admitting an essential conformal transformation, Uspekhi Mat. Nauk 28 (1973) 225-226, in Russian.

[BdM] L. Boutet de Monvel, Intégration des équations de Cauchy-Riemann induites formelles, in "Equations aux Dérivées Partielles Linéaires", Sém. Goulaouic-Lions-Schwartz, 1974-1975, Exposé 9.

[B] L. Bungart, Holomorphic functions with values in locally convex spaces and applications to integral formulas, Trans. Amer. Math. Soc. 111 (1964) 317-344.

[BS] D. Burns, Jr. and S. Shnider, Geometry of hypersurfaces and mapping theorems in $\mathbf{C}^{\mathbf{n}}$, Comm. Math. Helvetici 54 (1979) 199-217.

[CL] J.-H. Cheng and J. M. Lee, A local slice theorem for 3-dimensional CR structures, preprint, 1994.

[CM] S.-S. Chern and J. K. Moser, Real hypersurfaces in complex manifolds, Acta Math. 133 (1974) 219-271; Erratum, Acta Math. 150 (1983) 297.

[Fe] C. Fefferman, The Bergman kernel and biholomorphic mappings of pseudoconvex domains, Invent. Math. 26 (1974) 1-65.

[F1] J. (Lelong-)Ferrand, Transformations conformes et quasi-conformes des varietés riemanniennes, Acad. Roy. Belgique Sci. Mem. Coll. 839 (1971).

[F2] The action of conformal transformations on a riemannian manifold, preprint.

[FK] G. B. Folland and J. J. Kohn, "The Neumann Problem for the CauchyRiemann Complex", Princeton Univ. Press, Princeton, 1972.

[Fo] F. Forstnerič, An elementary proof of Fefferman's theorem, Exp. Math. 10 (1992) 135-149.

[Fu] H. Fujimoto, On the holomorphic automorphism groups of complex spaces, Nagoya Math. J. 33 (1968) 85-106.

[Gr] J. W. Gray, Some global properties of contact structures, Ann. Math. 69 (1959) 421-450.

[Gut] K. R. Gutschera, Invariant metrics for groups of conformal transformations, preprint, 1993.

[G1] R. C. Gunning, On Vitali's theorem for complex spaces with singularities, J. Math. Mech. 8 (1959) 133-141.

[G2] _ "Lectures on Complex Analytic Varieties: The Local Parametrization Theorem", Princeton Univ. Press, Princeton, 1970.

[GR] R. C. Gunning and H. Rossi, "Analytic Functions of Several Complex Variables", Prentice-Hall, Englewood Cliffs, 1965.

$[\mathrm{H}] \quad$ L. Hörmander, "An Introduction to Complex Analysis in Several Variables", North-Holland, New York, 1990.

[HL] R. Harvey and B. Lawson, On boundaries of complex analytic varieties I, Ann. of Math. 102 (1975) 233-290.

[Ko] S. Kobayashi, "Transformation Groups in Differential Geometry", Springer-Verlag, New York, 1972. 
[La] J. Lafontaine, The theorem of Lelong-Ferrand and Obata, in "Conformal Geometry", Aspects of Math. E, R. Kulkarni and M. Pinkall (eds.), MaxPlanck Inst. Für Math., Bonn (1988) 93-103.

[Le] J. M. Lee, Pseudo-Einstein structures on CR manifolds, Amer. J. Math. 110 (1988) 157-178.

[MZ] D. Montgomery and L. Zippin, Existence of subgroups isomorphic to the real numbers, Ann. of Math. (2) 53 (1951) 298-326.

[O] M. Obata, The conjectures on conformal transformations of Riemannian manifolds, J. Differential Geometry 6 (1971) 247-258.

[P] P. Pansu, Distances conformes et cohomologie $L^{n}$, Publ. Univ. Pierre et Marie Curie, 92 (1990).

[R] J.-P. Rosay, Sur une caractérisation de la boule parmi les domaines de $\mathbf{C}^{\mathbf{n}}$ par son groupe d'automorphismes, Ann. Inst. Fourier Grenoble 29, 4 (1979) 91-97.

[T] N. Tanaka, "A Differential Geometric Study on Strongly Pseudo-Convex Manifolds", Kinokuniya Company Ltd., Tokyo, 1975.

[Wo] B. Wong, Characterization of the unit ball in $\mathbf{C}^{\mathbf{n}}$ by its automorphism group, Invent. Math. 41 (1977) 253-257.

[We1] S. M. Webster, Real hypersurfaces in complex space, dissertation, University of California, Berkeley, 1975.

[We2] On the transformation group of a real hypersurface, Trans. Amer. Math. Soc. 231 (1977) 179-190.

[We3] , Pseudohermitian structures on a real hypersurface, J. Differential Geometry 13 (1978) 25-41.

VERSION 2.2 - MAY 2, 1994

Department of Mathematics, GN-50, University of Washington, SeatTLE, WA 98195

E-mail address: lee@math.washington.edu 